\title{
Analysis of Forces Involved in the Perching Maneuver of Flapping-Wing Aerial Systems and Development of an Ultra-Lightweight Perching System
}

\author{
V. Pérez-Sánchez*, A.E. Gómez-Tamm*, F.J. García-Rubiales*, B. Arrue*, A. Ollero*
}

\begin{abstract}
Trying to optimize the design of aerial robotics systems, this work presents an optimized low-weight landing system for flapping-wing aerial robots. The design, based on the use of low-sized neodymium magnets, intends to provide that these aerial robots have the capability of landing in restricted areas by using the presented solution. This capacity will increase the application range of these robots. A study of this situation has been done to analyze the perching maneuver forces and evaluate the system. The solution presented is low-weight, lowsized, and also relatively inexpensive. Therefore, this solution may apply to most ornithopter robots. Design, analysis of the implied forces, development and experimental validation of the idea are presented in this work, demonstrating that the developed solution can overcome the ornithopter's payload limitation providing an efficient and reliable solution.
\end{abstract}

Aerial Robotics, UAS, Flapping-Wings, Magnetic EndEffectors

\section{INTRODUCTION}

The idea of developing aerial robotics technologies for fulfilling different purposes has evolved in the last decades in various fields. With every successful application, the range of potential uses for these Unmanned Aerial Systems (UAS) has been extended.

Regarding visual surveillance applications, including manipulation and transportation or even co-working, are some fields where UAS apply. However, for applications involving confined spaces and interaction with humans or critical facilities, the risk that entails the use of a flying autonomous object of a significant weight can be considerable.

For that reason, the use of multirotors - mostly UAS in industrial applications, has been restricted due to the risk of crashing or harming with their blades. As an alternative, flapping-wing aerial systems or ornithopters are gaining importance.

These ornithopters offer an hybrid between the attributes of fixed wings aerial systems and multirotors. One of the most remarkable is the lack of blades or any kind of component that could be a potential danger for their environment while operating. This is due their motion using the force generated by their wings movement.

However, one of the main disadvantages is the minor maneuverability when compared with multirotors. This is more significant in takeoff and landing maneuvers. The first

\footnotetext{
${ }^{*}$ GRVC Robotics Laboratory, University of Seville. Avenida de los Descubrimientos S/N 41092 Spain vpsanchez@us.es, agtammeus.es, fragarrubealum.us.es, barruedus.es, aollerodus.es
}

one can be solved by using a launching system or a manual launch in the case of small or medium sized systems. It is the second one which implies more risks when performed.

Flapping-wing systems tend to crash or perform a not controlled landing maneuver while performing landing operations. This is due to a variety of reasons. The main reason is that they are not special landing gears for these type of UAS. The strict payload limitations of these systems make it difficult to create a reliable landing gear that does not disturb the flying operations that must be performed by the ornithopter.

For that reason, this work proposes the use of neodinium magnets integrated in a ultralight-weight landing gear to allow the flapping-wing aerial system to perform landing by using a simple magnetic landing platform.

By doing so, systems with this kind of landing system will be capable of performing landing in very restricted places, maintaining the security and safety needed.

Design geometries will take inspiration in nature but will focus on minimizing the weight added to the aerial platform while maximizing performance.

Design, development and experimental validation will be shown in this work to demonstrate the viability of performing landing with flapping-wing systems in a very restricted place.

The rest of the paper is structured as follows. Section II will focus on the summary of the state of the technologies presented in this work. Section III will present the landing system concept and design, as well as the development and the parts that compose the developed solution. Section IV will present the experiments performed in the developed setup and the result obtained. Finally, Section V will show the conclusions and potential future works.

\section{STATE OF ART}

Over the last decades, aerial systems have presented their potential for fulfilling various tasks. This can be confirmed in several previous works like [1] or [2] where there are different reviews about the appearance of specific aerial systems for different purposes.

The evolution from the military fixed-wings used for surveillance and data collection in previous years [3] had lead to the use of rotary-wing aerial systems used in civil applications for close-range inspection [4] and manipulation [5].

As first concept tries, helicopters were used like in [6] or [7] adding some manipulators and end-effectors to these 
systems. However, the use of these large-sized aerial systems implies a high risk to the surrounding environment, specially human beings.

Studies like [8], [9], [10] or [11] prove that technology has evolved to the use of medium and small-sized Unmmaned Aerial Vehicles (UAV), specially multi-rotors, and tries to maximize the system's performance by reducing the payload.

Even with all this progress, the risk of using this kind of aerial robotic systems is still considerable. The main ones are the existence of sharp and potentially dangerous mobile parts like the propellers, and also the intrinsic instability of the systems that make them uncontrollable in case of an engine failure. Some studies have been done [12] with the idea of mitigating the consequences of these events. However, zero risk seems not to be reachable by now.

When the risk is not assumable by other aerial platforms, flapping-wing systems could be an alternative solution for these applications [13], [14]. UAS apply the use of their wings to perform enough force to fly, so no propellers are implied. Moreover, in case of an engine failure, they can perform gliding for an emergency landing instead of an uncontrolled crash.

Some authors have studied the behaviour and performance of this kind of aerial systems [15]. Also, they have developed some add-ons or end-effectors [16] for these UAS, or even landing gears for medium sized multirotors [17]. This knowhow has helped to study the risky maneuver of performing landing so a potential solution can be proposed.

This problem concerns the use of this type of UAS and has been studied over the years [18], [19]. For these authors, there are no landing gears for flapping-wing systems due to the strict payload limitation. Previous solutions always tried to optimize the landing operation to allow a relatively safe crash on the ornithopter's belly. However, this operation also needs enough space. The design of an ultra-low weight landing gear using neodymium magnets [20], specially flappingwings aerial systems, could change this concept.

This kind of magnets have been used in several robotics applications over the years like [21] or [22]. Their unique attributes for performing high forces while maintaining a low-weight have high potential in aerial applications. For this reason, they have been used in different works as for object delivery [23], battery changing [24] or even perching of micro aerial systems [25].

For all mentioned above, the authors consider solutions presented in this work feasible and high-potential to improve the performance of the flapping-wing system.

\section{DESIGN AND DEVELOPMENT}

In this section, the developed system is presented and the different steps done for the design are described. Starting by analyzing the flight states, Figure 1 shows the different phases of an Ornithopter flight while approaching the landing platform showing the proposed solution. This paper is focused on the landing phase, also called the contact phase in this article. It will focus on analyzing the dynamics involved in the contact phase and will then propose a system to ensure a successful landing.

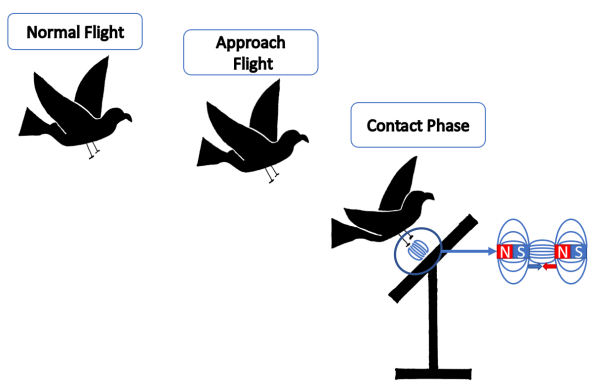

Fig. 1: Flight Phases involving the presented approach. The flapping wing system changes from a normal flight position to an approach flight to the landing platform and finally a contact phase, finishing the maneuver.

\section{A. IMPACT ANALYSIS}

This section is focused on the calculation of the theoretical forces implied in the impact. Two impact cases will be analyzed. For the first one, the aerial system does not have claws. Therefore, it resembles a system without damping. In the second case of study, the claws are incorporated and the stiffness and damping are taken into account.

The study considers the parameters from the ornithopter related to the impact. The first evaluated parameter is velocity. The ornithopter performing a proper flight can reach a speed from $4 \mathrm{~m} / \mathrm{s}$ to $10 \mathrm{~m} / \mathrm{s}$. In an approach phase, the flapping-wing should reduce the speed to $3 \mathrm{~m} / \mathrm{s}$. Apart from the velocity, the mass of the system is another important parameter to analyze in the impact. The study focus on an ornithopter of around $1,5 \mathrm{Kg}$ weight.

Regarding the impact forces, the system developed in this work will support the impact and also absorb the rebound forces. The free vibration equation is used involving the model of the system to calculate the impact forces. Initial considerations imply that no time-varying external forces act on the system. The theoretical system has one degree of freedom because its motion can be completely described by a single scalar variable. The system must be linear so that the equation of motion is also linear. This approach is based on the work developed in [26] and [27].

The first approach refers to the first case of study. In this approach, the system is an ornithopter without claws landing on the surface. The equation of motion used for this approach is based on an undamped spring-mass system:

$$
\begin{aligned}
& u_{c}(t)=\left(A \cos \omega_{n} t\right) \\
& \text { where: } \omega_{n}=\sqrt{k / m}
\end{aligned}
$$

Where $m$ is the mass system, $k$ is the stiffness, $u$ is the displacement and $A$ is the initial displacement $u_{0}$.

The second approach refers to the second case. The claws are incorporated on the platform and the equation of motion for a damped spring-mass system is used: 


$$
\begin{array}{r}
u_{c}(t)=e^{-\zeta \omega_{n} t}\left(A \cos \omega_{D} t+B \sin \omega_{D} t\right) \\
\text { where: } \quad \begin{aligned}
\omega_{D} & =\omega_{n} \sqrt{1-\zeta^{2}} \\
B & =\frac{\dot{u}_{0}+\zeta \omega_{n} u_{0}}{\omega_{D}}
\end{aligned}
\end{array}
$$

Where $m$ is the mass, $k$ is the stiffness ratio, $c$ is the damping coefficient, $\zeta$ is the damping ratio, $u$ is the displacement, $\dot{u}$ is he velocity, $\ddot{u}$ is the acceleration and $\mathrm{A}$ is the initial displacement $u_{0}$.

In this stage, the rebound speed is calculated. A coefficient of restitution $e$ of 0.8 is supposed. This implies an impact near to a perfectly elastic collision but assuming that the materials of the surface and the claws materials can partially absorb the collision's force. The rebound speed will be obtained by applying the restitution coefficient equation and the momentum equation.

$$
\left\{\begin{array}{l}
m_{a p} \cdot\left(v_{a p}^{+}-v_{a p}^{-}\right)=I_{c} \\
m_{p p} \cdot\left(v_{p p}^{+}-v_{p p}^{-}\right)=-I_{c} \\
e=-\frac{v_{a p}^{+}-v_{p p}^{+}}{v_{a p}^{-}-v_{p p}^{-}}
\end{array}\right.
$$

Where $m_{a p}$ is the mass of the aerial platform, $v_{a p}^{+}$is the velocity of the aerial platform after the impact, the variable that must be calculated, $v_{a p}^{-}$is the velocity before the impact of the aerial platform, this speed is assumed to be $3 \mathrm{~m} / \mathrm{s}$ and $I_{c}$ the momentum. The mass of the perching platform is $m_{p p}, v_{a p}^{-}$is the velocity before the impact of the perching platform and $v_{p p}^{+}$is the velocity of the perching platform after the impact.

Lastly, the system is solved. The velocities after the impact on the perching and aerial platform are known.

The last step is to calculate the model stiffness $k$. This is the last parameter needed to calculate the equilibrium equation.

$$
k=\frac{E \cdot A}{L}
$$

Where $\mathrm{E}$ is the Young module, $\mathrm{A}$ is the area of a fibre carbon tube and $\mathrm{L}$ is the length.

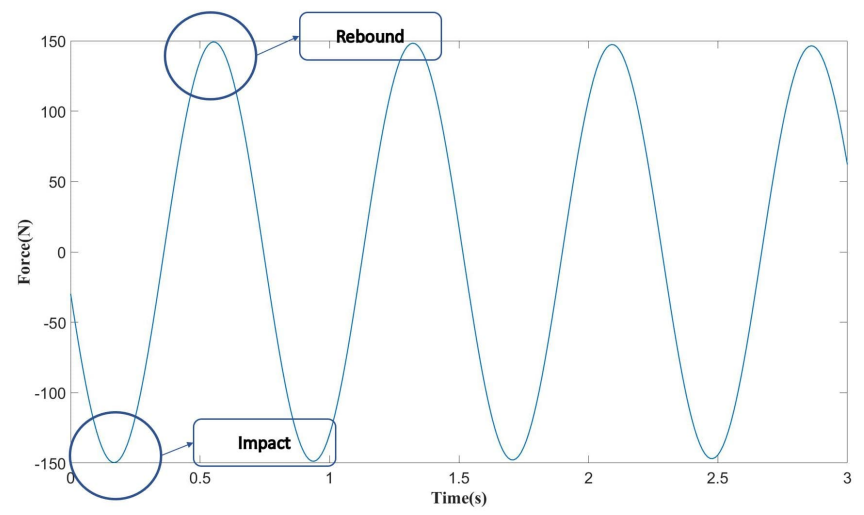

Fig. 2: Impact Forces results. A sine wave is obtained where the maximum and minimum values of the graphic shows the maximum forces applied to the system when impacting.
Figure 2 shows the theoretical force results for the first case of study. The main part of this graph is the two first peaks. The first one refers to the impact and the second peak specifies the rebound force. The rest of the graphics is for the vibratory situation not analyzed in this paper. The lack of a dumper makes the impact and rebound force equals. This work is interested in the reduction of the rebound force to make possible the perch with an ultra light-weight system.

The second case of study will be shown and analyzed in Section III-B Figure 5 using the properties of the system developed.

\section{B. SYSTEM RESTRICTION}

This subsection will focus on the restrictions added by the intrinsic characteristics of the aerial platform to the proposed system. There are two main restrictions: the payload and the angle in the contact phase.

The first restriction affects directly the magnetic claws and is the main reason to develop the system presented in this work. Ornithopters are low-weight aerial platforms and the system payload capacity is only around 300 gr. These numbers do not take into account the flight electronics and the power supply of the system. These limitations are a big problem to integrate different systems in the same platform. For example, the weight of a low-weight camera with an embedded computer to control it is around $200 \mathrm{gr}$. The rest of the systems should then be integrated into a $100 \mathrm{gr}$ weight.

Taking this restriction into account, proposed solutions must have as little mass as possible. For this reason, the system developed in this work avoids any external actuation, which would include electronics and system power supply.

The system developed has to be also as simple as possible. The reduction of mass can make the system smoother. This could be a problem, because the system has to resist high forces as seen in Figure 2.

On the other hand, the restriction on the landing angle affects the claws and landing platform systems and the relation between them. The ornithopters cannot fly in all the inclination angles. For this reason, the systems developed have to overcome this restriction.

\section{SYSTEM OVERVIEW}

This subsection presents and analyzes the systems developed in this work. The system takes into consideration the restrictions and forces calculations presented in the previous sections.

Firstly, this section focus on the development of the claws. Figure 3 shows an overview of the claws.

The claws developed in this work are composed of different parts contact end-effector, legs, links, and dumper. Each one of these parts has different functionalities and are made of different materials. The complete system solves the restrictions presented before.

This system provides contact to the landing platform and makes it possible to maintain the equilibrium in the longitudinal direction. Several tests have been done to analyze the weaknesses of this end-effector. 


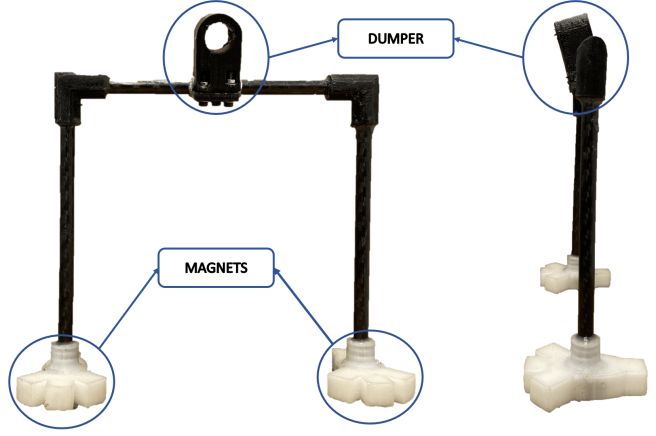

Fig. 3: Developed magnetic Claws Overview. It is divided into three main parts dumper, frame and claws.

The first step was to decide the end-effector material. Several tests were done to select the material, starting with 3D printed Polylactic Acid (PLA). The experiments show this material as brittle for this kind of use. Finally, 3D printed Nylon was selected. Its elasticity combined with its hardness and low-weight was the reason to use this material in the end-effector.

The next step was to select the geometry of this actuator. The inclusion of two claws guarantees equilibrium when the system is perching. However, the equilibrium in the longitudinal axis is not guaranteed. The solution was to generate a geometry with a major surface on the longitudinal axis. Before the fatigue test, a weakness point was detected in the link between the end-effector and the leg. This was resolved by modifying the geometry of this link.

Legs are made of carbon fiber. This material is perfect for this system due to its low-weight high-resistance ratio. This part of the system has to communicate the impact forces to the dumper and links to absorb it.

Links are made of 3D printed Thermoplastic Polyurethane (TPU). A rigid system with low forces and flexibility can be developed depending on this material's density. The main objective of this system is to resist the high-impact forces maintaining the integrity of the whole claws system. Before the impact, the system has maintained the position to guarantee equilibrium.

The dumper is the last subsystem in the claws. The aim of this is to isolate the impact forces from the claws to the ornithopter and also to connect the entire system to the aerial platform. The dumper is made of Thermoplastic Polyurethane (TPU) in a different density configuration to meet the requirement of this part of the claws.

Table I shows the properties of the materials used for the development of the claws.

\begin{tabular}{ccccc}
\hline Material & $\begin{array}{c}\mathrm{E} \\
(\mathrm{MPa})\end{array}$ & $\begin{array}{c}\mathrm{G} \\
(\mathrm{MPa})\end{array}$ & $\begin{array}{c}\mathrm{e} \\
\%\end{array}$ & $\begin{array}{c}\text { Impact Resistance } \\
\left(\mathrm{KJ} / \mathrm{m}^{2}\right)\end{array}$ \\
\hline Carbon Fiber[28] & $58 \cdot 10^{3}$ & $3.6 \cdot 10^{3}$ & 42 & - \\
PLA [29] & 2346.5 & 3150 & 5.2 & 5.1 \\
Nylon [29] & 579 & 463.5 & 210 & 34.4 \\
TPU [29] & 26 & 78.7 & 580 & 34.4 \\
\hline
\end{tabular}

TABLE I: Materials properties
The table shows the differences between each material. In the case of absorber elements, the material selected is the one that has more impact resistance and elongation. Due to its high Yield strength and Shear modulus, carbon fiber was used to transmit the forces. In the case of the end effector, the data presented in the table confirm the result of the fatigue tests. For this purpose, Nylon was selected due to its high impact resistance when comparing with PLA.

The claws developed have a reduced surface when comparing with the ornithopter aerodynamics surfaces. This makes it possible to reduce the aerodynamics disturbances of the system.

Several fatigue tests have been done on all the components. The next step is to calculate the needed strength to perch in the landing platform by applying the methods presented in Section III-A. Particularly, Figure 4 shows a representation of the model implemented to develop the theoretical calculations.

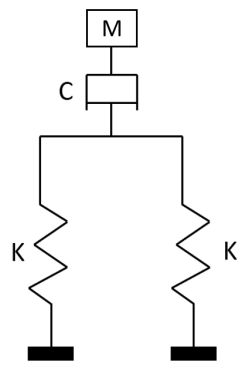

Fig. 4: Simplified diagram of the magnetic claws

In Figure 4, K represents the stiffness for each leg of the system, $\mathrm{C}$ represents the dumper of the system made with TPU and $\mathrm{M}$ is the mass of the ornithopter. The movement equation is showed in Equation 5.

$$
m_{a p} \cdot \ddot{u}(t)+C \cdot \dot{u}(t)+2 K \cdot u(t)=-m g \cdot \cos \alpha
$$

According to that, Figure 5 shows the theoretical results of the model.

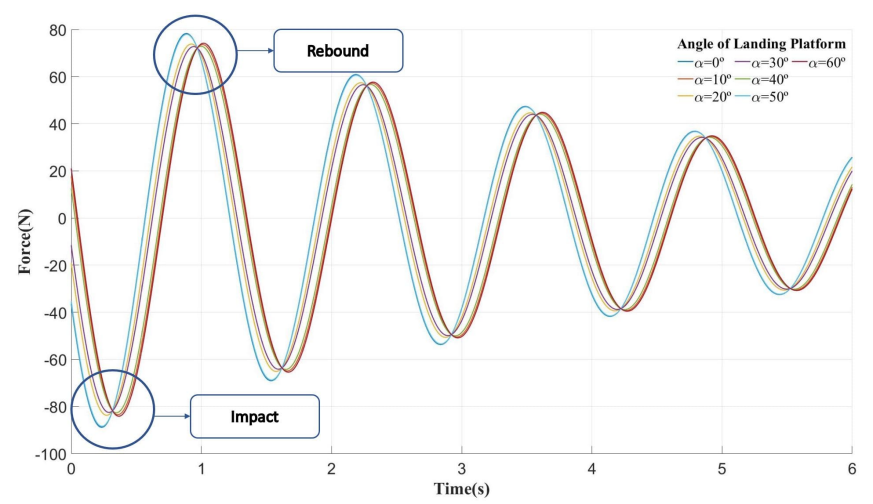

Fig. 5: Flight impact phase. The graphic presents the forces applied to the system using the proposed approaches 
Figure 5 shows the transitivity force in the function of the angle of the landing surface. The main parts of the graphic are the first and the second peak. The first one refers to the impact forces of the system and the second one refers to the rebound. The developed system has to resist the impact forces and absorb the rebound forces.

When comparing this graphic with the graphic of the initial state presented in Section III-A, the impact and rebound forces of the system are reduced in a percentage near to $50 \%$. The impact forces are reduced by the stiffness of the claws and the rebound forces are reduced by using the damping ratio of the system.

This graphic is crucial to evaluate the magnet needed to guarantee the perching. The magnet has to support the rebound forces of the system. Regarding Figure 5, the rebound forces are around 80 Newtons. The magnet is a commercial solution made of neodymium and it was selected due to its low-weight and high-force relation. The magnet has an attraction power of $60 \mathrm{~N}$. However, when using a magnet in each end-effector, the total force is around $120 \mathrm{~N}$.

The graphic shows the significance of the landing surface angle. An angle between 20 and 30 degrees is ideal to minimize the transmitted force. Also, the gliding angle has to be considered as seen in Section III-B. The best option for landing is to reach the surface gliding to reduce the contact velocity. Normally, ornithopters have a gliding between 10 and 20 degrees as seen in [15]. Regarding this, several experiments have been done using inclination surfaces between 10 and 30 degrees.

At last, the complete claws system has a weight of $35 \mathrm{gr}$ meeting the restriction of mass imposed in Section III-B. This result provides an improvement when compared with previous solutions and makes it possible to integrate this system with another functionality on the ornithopter.

Figure 6 shows the perching platform detailing the landing angle developed in this work to prove the proper operation of the system.

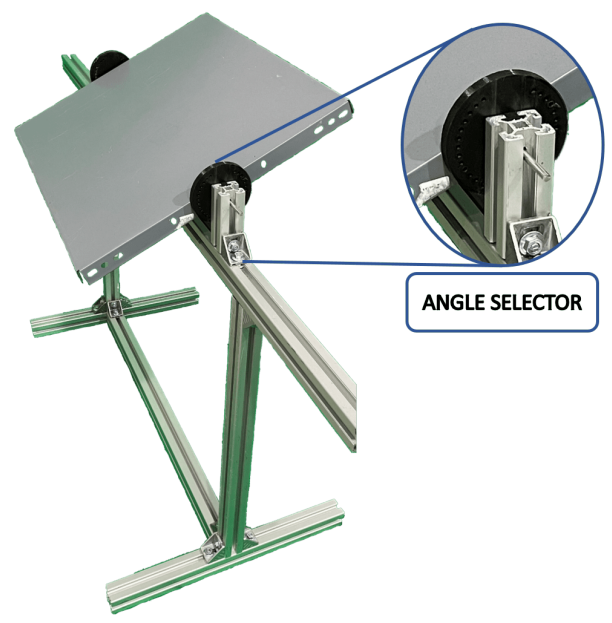

Fig. 6: Developed Perching Platform, the design improves success landing ratio.

The objective of the platform presented in the previous
Figure is to guarantee a wide range of experiments to analyze the behavior of the claws. This system could be used in situations where the trajectory of the ornithopter operation is defined. The capacity to select the landing angle is important not only for adjusting to the ornithopter trajectory but also for adapting to different surfaces and positions. The platform guarantees high accuracy in this situation as can be shown in Section IV-B.

\section{EXPERIMENTAL VALIDATION}

This section will focus on the experiments performed. The main objective is to prove the functionalities of the claws. Two kinds of experiments are showed. Firstly, a proof of the feasibility of the developed magnetic claws, and secondly, real tests were performed on a flight for validating the performance.

\section{A. Feasibility Test}

This section presents and analyzes the feasibility test. Tests were performed indoors. The aerial vehicle used is an ornithopter developed for the GRIFFIN ERC Advanced Grant project. To prove the actuation of the claws, the ornithopter is attached to a rope. The perching platform is a ferromagnetic rod. In this experiment, the landing accuracy was provided by the rope attached to the ornithopter. In this way, the system and actuation will be characterized by avoiding any possible inferences produced by other components presented in the ornithopter.

Figures 7, 8 and 9 show the results of these experiment. Figure 7 is a composition that includes the ornithopter approach to the rod and finally the contact phase with the magnets.

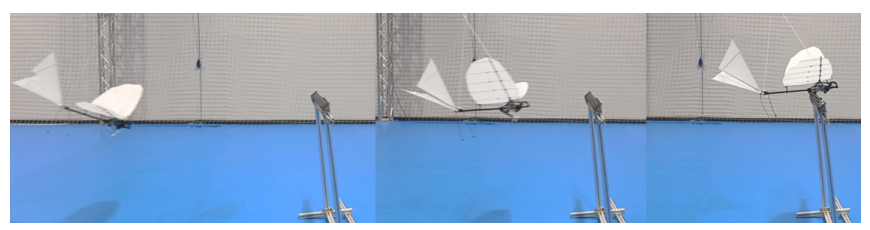

Fig. 7: Feasibility Test Lateral View

Figure 8 shows the ornithopter contact phase and the absorption of the impact forces by the claws in detail.

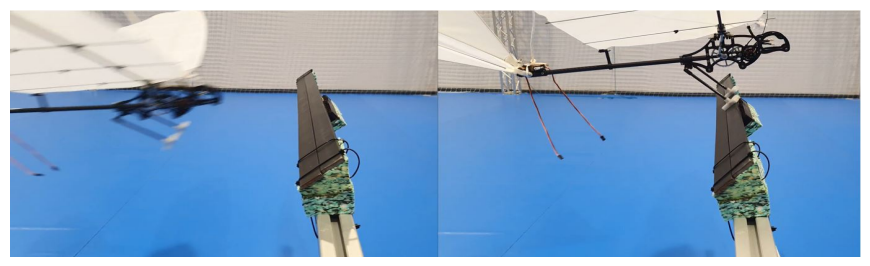

Fig. 8: Feasibility Test Detailed View

To analyze possible deformations in the longitudinal direction of the system, Figure 9 shows the contact phase from a bottom view.

In these experiments, the velocity reached was from 0.5 $\mathrm{m} / \mathrm{s}$ to $2 \mathrm{~m} / \mathrm{s}$. The results were excellent. The claws were able 


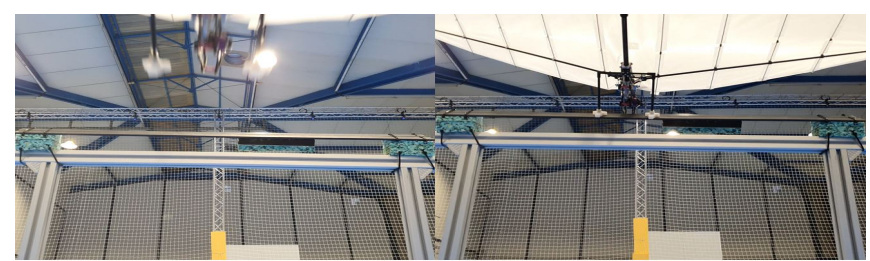

Fig. 9: Feasibility Test Bottom View

to support and absorb the forces generated in the impact. The rigid structure of the claws combined with soft links worked properly. In figure 7, different positions, before and after the impact, are shown. This situation makes it possible to absorb the impact forces and isolate them from the rest of the aerial vehicle. Furthermore, the claws perching on the rod support the impact forces and the moment produced by the rope.

\section{B. Real Flight Test}

This section shows the results of free flight experiments. The aerial vehicle used in these experiments is the same that the one used in Section IV-A. The main difference in the setup of the ornithopter is the inclusion of all systems and electronics needed to fly. Another difference between these experiments and the previous one is the use of the landing platform designed in this work. A wide range of landing platform angles has been evaluated. The angle selected for the perching in this platform is the one that reduced the impact forces making it possible for the ornithopter to perform a stable flight.

Figure 10 shows the approaching deviations absorbed by the landing platform.

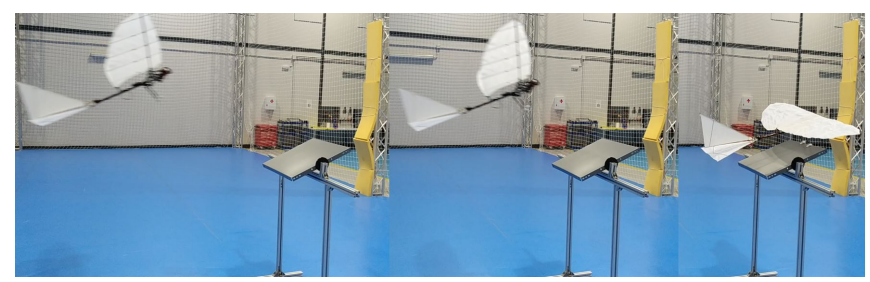

Fig. 10: Perching approach adjustments performed by the magnets magnetic field forces

In this figure, accuracy demonstration provided by the landing platform is shown. A wide range of experiments has been done perching in different parts of the landing platform.

Figure 11 shows a landing in the center of the landing platform.

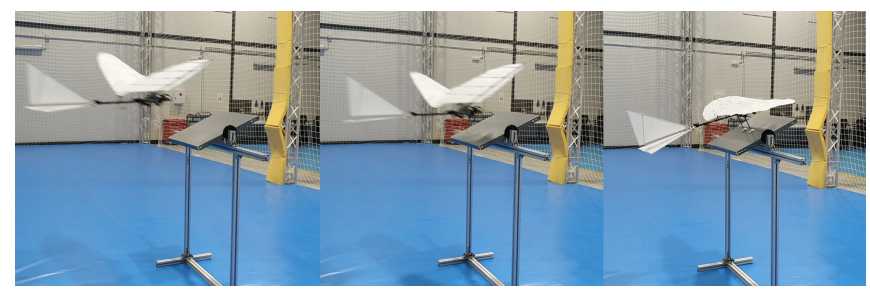

Fig. 11: Land on the perching surface without deviations
In this test, a little magnet was attached to the center of the landing platform surface. This magnet increases the magnetic field of this place improving the accuracy of this experiment and showing the result presented in Figure 11.

To prove the proper system's operation, the ornithopter was launched in a gliding flight reaching a speed of $5 \mathrm{~m} / \mathrm{s}$. Claws have demonstrated to work properly in more exigency conditions than the estimated in a normal flight. The landing platform accommodates the gliding angle of the system by using the angle selector developed in the platform. This system has been proved in several successful experiments to analyze the fatigue strength of the claws. Results of the fatigue experiments were important to improve the system to the current version.

At last, after the experiments, the model developed integrated into the aerial platform is shown in Figure 12.

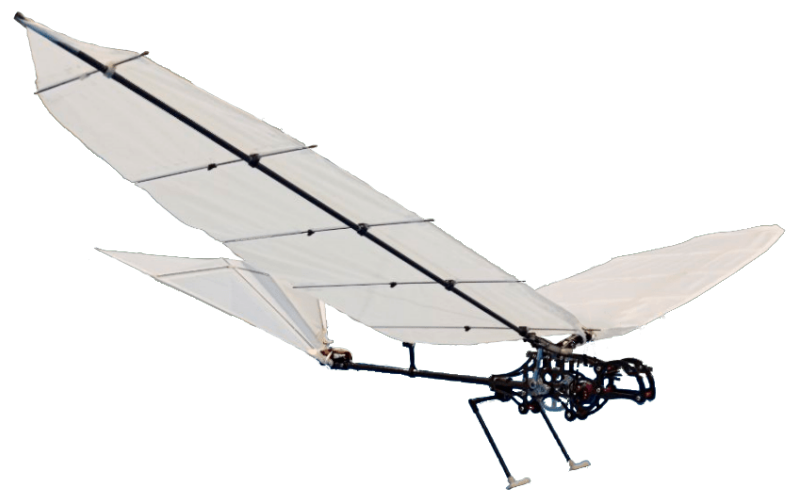

Fig. 12: Final Developed Model

It is proved that this model can perch on the landing platform with velocities up to $5 \mathrm{~m} / \mathrm{s}$. Claws can absorb a part of the impact maintaining the integrity of the system. Regarding the graphic presented in Section III-C and taking into consideration the gliding angel of the ornithopter, the angle selected in the platform is 20 degrees. This angle reduced the impact forces maintaining a proper angle on the ornithopter approach phase. Both systems, claws and landing platform, work properly. However, it is possible to perch by only using the claws as seen in Section IV-A.

\section{CONCLUSIONS}

In this work, a study of the perching forces and an improvement for the capacity of ornithopters to perform landing or perching operations are presented. The developed system is named an ultralow-weight perching system.

It has been proved that the landing system can overcome the intrinsic payload limitations of flapping-wing aerial systems. Due to the simplicity in the actuation and novelty design, the developed product is robust and reliable and allows a high success ratio in the landing operation.

Results of this work also show the capacity of the system to absorb the impact generated in the operation. They also prove its capability to reduce any potential damage that UAS could receive. Also, the approach makes it possible for the ornithopter to land in very constricted areas. 
Regarding these results, the perching forces study shows a proper estimation of the forces to evaluate the system.

The proposal solution developed is scalable and adaptable to different Aerials platforms providing a new benchmark for perching applications.

Future works will focus on combining this technology with systems, such as vision sensors, that could be embedded in the ornithopter for fulfilling more complex tasks.

Successful perchings could improve by using little neodymium magnets on the platform surface. These magnets increase the magnetic field, hence the attraction range between platforms and claws. However, impact forces will increase.

The developed system is intended to be used in surveillance applications, like natural disasters, offering a reliable autonomous high-range system with the capacity of landing in constricted areas.

\section{ACKNOWLEDGMENT}

We thank Robotics, Vision and Control Group for supporting us during this work. This work has been developed under the framework of the project GRIFFIN (General compliant aerial Robotic manipulation system Integrating Fixed and Flapping-wings to INcrease range and safety) SI-1867/23/2018 ERC-ADG - Advanced Grant EU-funded project.

\section{REFERENCES}

[1] Fabio Ruggiero, Vincenzo Lippiello, and Anibal Ollero. Aerial manipulation: A literature review. IEEE Robotics and Automation Letters, 3(3):1957-1964, 2018.

[2] Hossein Bonyan Khamseh, Farrokh Janabi-Sharifi, and Abdelkader Abdessameud. Aerial manipulation-a literature survey. Robotics and Autonomous Systems, 107:221-235, 2018.

[3] David H Lyon. A military perspective on small unmanned aerial vehicles. IEEE Instrumentation \& Measurement Magazine, 7(3):2731, 2004.

[4] Roseneia Rodrigues Santos De Melo, Dayana Bastos Costa, Juliana Sampaio Álvares, and Javier Irizarry. Applicability of unmanned aerial system (uas) for safety inspection on construction sites. Safety science, 98:174-185, 2017.

[5] Felipe R Fabresse, Fernando Caballero, Ivan Maza, and Anibal Ollero. Localization and mapping for aerial manipulation based on rangeonly measurements and visual markers. In 2014 IEEE international conference on robotics and automation (ICRA), pages 2100-2106. IEEE, 2014.

[6] Konstantin Kondak, Felix Huber, Marc Schwarzbach, Maximilian Laiacker, Dominik Sommer, Manuel Bejar, and Aníbal Ollero. Aerial manipulation robot composed of an autonomous helicopter and a 7 degrees of freedom industrial manipulator. In 2014 IEEE international conference on robotics and automation (ICRA), pages 2107-2112. IEEE, 2014.

[7] Paul EI Pounds, Daniel R Bersak, and Aaron M Dollar. The yale aerial manipulator: grasping in flight. In 2011 IEEE International Conference on Robotics and Automation, pages 2974-2975. IEEE, 2011.

[8] Arvid QL Keemink, Matteo Fumagalli, Stefano Stramigioli, and Raffaella Carloni. Mechanical design of a manipulation system for unmanned aerial vehicles. In 2012 IEEE international conference on robotics and automation, pages 3147-3152. IEEE, 2012.

[9] Suseong Kim, Seungwon Choi, and H Jin Kim. Aerial manipulation using a quadrotor with a two dof robotic arm. In 2013 IEEE/RSJ International Conference on Intelligent Robots and Systems, pages 4990-4995. IEEE, 2013.
[10] Carmine Dario Bellicoso, Luca Rosario Buonocore, Vincenzo Lippiello, and Bruno Siciliano. Design, modeling and control of a 5dof light-weight robot arm for aerial manipulation. In 2015 23rd Mediterranean Conference on Control and Automation (MED), pages 853-858. IEEE, 2015.

[11] A Suarez, AE Jimenez-Cano, VM Vega, G Heredia, A RodriguezCastaño, and A Ollero. Lightweight and human-size dual arm aerial manipulator. In 2017 International Conference on Unmanned Aircraft Systems (ICUAS), pages 1778-1784. IEEE, 2017.

[12] Lina Castano and Huan Xu. Safe decision making for risk mitigation of uas. In 2019 International Conference on Unmanned Aircraft Systems (ICUAS), pages 1326-1335. IEEE, 2019.

[13] Derrick Yeo, Ella Atkins, Luis Bernal, and Wei Shyy. Experimental investigation of the pressure, force, and torque characteristics of a rigid flapping wing. In 50th AIAA Aerospace Sciences Meeting including the New Horizons Forum and Aerospace Exposition, page 849, 2012.

[14] Wei He, Haifeng Huang, Yunan Chen, Wenzhen Xie, Fusen Feng, Yemeng Kang, and Changyin Sun. Development of an autonomous flapping-wing aerial vehicle. Science China Information Sciences, 60(6):063201, 2017.

[15] R Lopez-Lopez, V Perez-Sanchez, P Ramon-Soria, A MartínAlcántara, R Fernandez-Feria, BC Arrue, and A Ollero. A linearized model for an ornithopter in gliding flight: Experiments and simulations. In 2020 IEEE International Conference on Robotics and Automation (ICRA), pages 7008-7014. IEEE, 2020.

[16] AE Gomez-Tamm, V Perez-Sanchez, BC Arrue, and A Ollero. Sma actuated low-weight bio-inspired claws for grasping and perching using flapping wing aerial systems. In Proceedings of the 2020 IEEE/RSJ International Conference on Intelligent Robots and Systems (IROS), Las Vegas, NV, USA, pages 25-29, 2020.

[17] P Ramon-Soria, Alejandro Ernesto Gomez-Tamm, FJ Garcia-Rubiales, Begoña C Arrue, and Aníbal Ollero. Autonomous landing on pipes using soft gripper for inspection and maintenance in outdoor environments. In 2019 IEEE/RSJ International Conference on Intelligent Robots and Systems (IROS), pages 5832-5839. IEEE, 2019.

[18] Aditya A Paranjape, Soon-Jo Chung, and Joseph Kim. Novel dihedralbased control of flapping-wing aircraft with application to perching. IEEE Transactions on Robotics, 29(5):1071-1084, 2013.

[19] Si Chen, Shijun Guo, Hao Li, Mingbo Tong, and Bing Ji. Short landing performance and scale effect of a flapping wing aircraft. Journal of Aerospace Engineering, 33(6):04020085, 2020.

[20] JJ Croat. Current status and future outlook for bonded neodymium permanent magnets. Journal of applied physics, 81(8):4804-4809, 1997.

[21] Brian Bridge, Jianzhong Shang, Bryan Bridge, Tariq Sattar, Shyamal Mondal, and Alina Brenner. Development of a climbing robot for inspection of long weld lines. Industrial Robot: An International Journal, 2008.

[22] K-M Lee and C-K Kwan. Design concept development of a spherical stepper for robotic applications. IEEE Transactions on Robotics and Automation, 7(1):175-181, 1991.

[23] Abel Gawel, Mina Kamel, Tonci Novkovic, Jakob Widauer, Dominik Schindler, Benjamin Pfyffer Von Altishofen, Roland Siegwart, and Juan Nieto. Aerial picking and delivery of magnetic objects with mavs. In 2017 IEEE international conference on robotics and automation (ICRA), pages 5746-5752. IEEE, 2017.

[24] Koji AO Suzuki, Paulo Kemper Filho, and James R Morrison. Automatic battery replacement system for uavs: Analysis and design. Journal of Intelligent \& Robotic Systems, 65(1):563-586, 2012.

[25] K Zhang, Pisak Chermprayong, TM Alhinai, Robert Siddall, and Mirko Kovac. Spidermav: Perching and stabilizing micro aerial vehicles with bio-inspired tensile anchoring systems. In 2017 IEEE/RSJ International Conference on Intelligent Robots and Systems (IROS), pages 6849-6854. IEEE, 2017.

[26] Dominguez Abascal J Mayo Nunez JM. Mecaica de mauinas y vibraciones: vibraciones de 1 gdl. 2015.

[27] Mayo Nunez JM. JM, Dominguez Abascal J. Amp. teor y tecn. de muinas y mecanismos:problemas resueltos. 2015.

[28] FJ Garzón Lucena, C López Taboada, G Castillo López, and F García Sánchez. Design and manufacture of a self-supporting racing motorcycle seat made on carbon fiber. Materiales Compuestos, 2(2):34-39, 2018.

[29] Filaments properties. https://ultimaker.com/es/materials. Accessed: 2021-02-25. 